LBNL-62728

\title{
The Integration of Energy Efficiency, Renewable Energy, Demand Response and Climate Change: Challenges and Opportunities for Evaluators and Planners
}

\author{
Edward Vine \\ Environmental Energy Technologies Division \\ Building 90-4000 \\ Lawrence Berkeley National Laboratory \\ Berkeley, CA 94720
}

June 2007

The work described in this paper was funded by the Office of Energy Efficiency and Renewable Energy, Office of Planning, Budget and Analysis of the U.S. Department of Energy under Contract No. DEAC02-05CH11231 



\begin{abstract}
This paper explores the feasibility of integrating energy efficiency program evaluation with the emerging need for the evaluation of programs from different “energy cultures” (demand response, renewable energy, and climate change). The paper reviews key features and information needs of the energy cultures and critically reviews the opportunities and challenges associated with integrating these with energy efficiency program evaluation. There is a need to integrate the different policy arenas where energy efficiency, demand response, and climate change programs are developed, and there are positive signs that this integration is starting to occur.
\end{abstract}




\section{Introduction}

The evaluation landscape is changing for evaluators and planners of energy efficiency (EE) programs. Historically, policymakers and utility regulators have considered EE as the least cost strategy to help meet resource adequacy and transmission expansion needs (especially in geographically targeted congested areas) and to mitigate increasing energy costs. Recently, state and federal policymakers and utility regulators have broadened the scope of evaluation and planning by integrating EE programs focused on achieving energy savings with programs that focus on other program objectives such as reducing dependency on fossil fuels (renewable energy), reducing the need for investments in generating capacity (demand response), and investing in technologies that help to mitigate climate change (Michals and Titus 2006). ${ }^{1}$ As a result, the following types of questions are being asked of evaluators and planners:

1. How can the evaluation of EE programs provide guidance on the design and evaluation of these other types of programs? Specifically, what evaluation issues are similar and what are different?

2. What EE program evaluation approaches are useful for evaluating renewable energy, demand response, and climate change mitigation programs?

3. How are state evaluation protocols developed for evaluating EE programs being expanded to include other topics and being extended regionally and nationally?

4. What policy mechanisms are needed for integrating EE programs with these other types of programs?

In this paper, we first examine the changing landscape and then discuss several key evaluation issues resulting from the evaluation of EE programs that will be relevant for the evaluation of DR, RE, and CC programs. We next focus on those evaluation approaches and methods that will be helpful for evaluating the impacts from DR, RE, and CC programs and for program improvement. We then examine how current evaluation protocols are being expanded

${ }^{1}$ Energy efficiency is increasingly being interconnected (coordinated and integrated) with other areas that are not covered in this paper: for example, water efficiency, distributed generation, green buildings, LEED buildings, and sustainable communities. 
and extended. Finally, we describe the policy dilemma facing policy makers in integrating the different policy arenas and then provide examples of how integration is starting to occur.

\section{The Changing Landscape}

Energy efficiency is seen by many policymakers and utility regulators as the least cost strategy to help meet resource adequacy and transmission expansion needs (especially in geographically targeted congested areas). Since the energy crisis of 2000-2001 in California, multiple policy decisions have made EE the first priority electric resource (Vine et al. 2007). Since that time, California policymakers and utility regulators have established EE goals, policies, and programs. For example, in 2002, the California Public Utilities Commission (CPUC) required (Decision 02-10-062) that California's three electric investor-owned utilities (IOUs) follow a specific resource loading order when developing their integrated resource plans and searching for new energy supplies, stating that resource adequacy needs should first be met through all cost-effective EE and demand-response programs.

California was not the only state in the West that was interested in promoting EE in the aftermath of the consumer price shocks and short-term power shortages of the 2000-2001 electricity crisis. For example, the Northwest Power and Conservation Council (NPCC) established EE targets based on cost-effective EE potential for the Pacific Northwest region, and its $5^{\text {th }}$ Regional Power Plan is considered one of the best integrated resource plans in the country (NPCC 2005). Several state regulatory authorities in the Southwest (Arizona, Nevada, Utah) approved increases in utility EE spending (Geller 2006). At the regional level, the Western Governor's Association (WGA) has set a high priority for EE, establishing a goal of reducing projected electricity demand in 2020 by 20\% in a policy resolution on Clean and Diversified Energy for the West (WGA 2004). And a review of recent resource plans ${ }^{2}$ issued by fourteen investor-owned utilities in the Western United States and Canada found that four utilities had proposed EE programs that were projected to offset more than $70 \%$ of their forecasted energy

\footnotetext{
${ }^{2}$ Resource plans encompass integrated resource plans, default supply plans, long-term procurement plans, least-cost resource plans, and electric supply plans.
} 
load growth between 2004 and 2013 (Hopper et al. 2006). For these utilities, these programs were expected to reduce annual energy load growth from $1.6-2.6 \%$ per year to $\sim 0.5 \%$ or less. ${ }^{3}$

Energy efficiency is also seen as a solution for addressing transmission and distribution congestion problems on the grid. As with electricity generation, EE and other demand-side resources can be a cost-effective way to defer or eliminate the need for T\&D expansion (Kushler et al. 2005; Vine et al. 2007). Several electric system jurisdictions are actively pursuing such demand-side resources to address T\&D needs in the Pacific Northwest, New York, Vermont, Connecticut, and the City of San Francisco.

In addition to EE programs, demand response programs are getting increased attention for helping to solve the above problems. Demand response (DR) programs are designed to reduce short-term capacity needs and/or transmission constraints. DR is defined as changes in electricity consumption by customers in response to signals in the form of electricity prices, incentives, or alerts during periods when the electricity system is vulnerable to extremely high prices or compromises to reliability (CPUC 2007). In California, DR programs are an essential element of California's resource strategy, as articulated in the State's Energy Action Plan II (EAP II). EAP II ranks DR programs second in the "loading order" after energy efficiency programs and directs the utilities to subscribe at least $5 \%$ of system peak demand to DR programs by 2007. In 2006, the CPUC adopted price-responsive DR programs for large customers and set annual participation goals for utility DR programs (D. 03-06-032), which was subsequently followed by another decision (D. 06-03-024) where the CPUC approved \$262 million for DR programs for the years 2006-2008. And in its most recent DR decision (D. 06-11049), the CPUC authorized augmentations and expansions to the DR programs for the purposes of increasing DR as a resource for summer 2007. DR programs have become a focal point of the California Independent System Operator's (CAISO) wholesale market development. On Sept. 21, 2006, the Federal Energy Regulatory Commission (FERC) conditionally approved the CAISO’s Market Redesign and Technology Upgrade (MRTU) proposal and directed the CAISO to incorporate price-responsive DR programs in the MRTU. The CAISO plans to implement the day-ahead market of its MRTU during the first quarter of 2008. Other states in the U.S. (e.g., in

\footnotetext{
${ }^{3}$ The other utilities projected a more moderate role for energy-efficiency programs: e.g., four other utilities expected energy efficiency programs to offset 30 to $50 \%$ of forecasted load growth. And for three utilities, energyefficiency programs proposed in the resource plans were expected to play a very minor role $(0 \%$ to $7 \%$ of projected load growth).
} 
New England and New York) have also been very active in enacting DR policies and supporting DR programs.

Interest in renewable energy (RE) programs has been growing, and has been stimulated by the adoption of renewable portfolio standards (RPS) by 21 states in the U.S., along with the District of Columbia (Chen et al. 2007). ${ }^{4}$ A RPS requires that a minimum amount of RE is included in each retail electricity supplier's portfolio of electricity resources. For example, in California, Senate Bill (SB) 1078, passed in 2002, established a RPS and set a goal of renewable generation for $20 \%$ of the state's requirements by 2020. And in 2003, the CPUC and the CEC (via the first Energy Action Plan) accelerated SB 1078's RPS goal, moving the 20\% goal up to 2010. In September 2006, SB107 was signed into law and formally adopted this accelerated goal. Finally, Governor Schwarzenegger has proposed an even more ambitious goal of 33\% of renewable electricity sales by 2020. Also, in August 2006, Governor Schwarzenegger signed SB 1, which directed the CPUC and the CEC to implement the California Solar Initiative (CSI) program for the period 2007-2016. The overall goal of the CSI is to help lower the cost of solar systems for consumers and build a self-sustaining solar market. The CPUC, through the CSI, will be providing more than $\$ 2$ billion in incentives over the next decade for existing residential homes and existing and new commercial, industrial, and agricultural properties. The CEC is also managing a ten-year, \$350 million program to encourage solar installations in new home construction through its New Solar Homes Partnership (NSHP). A very important feature of the NSHP is that builders are required to exceed the current State's EE standards' (Title 24) performance by $15 \%$.

Interest in, and awareness of, potential climate change (CC) impacts (globally, regionally, and locally) are at an all-time high in the United States. Scientific studies (e.g., the Intergovernmental Panel on Climate Change’s recent report - IPCC 2007), changes in political power (e.g., the Democratic Party assuming control of the U.S. Senate and House), and the popular media (e.g., television, newspapers, books and movies (such as Al Gore's An Inconvenient Truth)) have all contributed to making "climate change” an important and urgent issue in the policy arena. Efforts to significantly reduce carbon emissions as part of any national strategy to address CC will require considerable improvements in EE. Studies by the National

\footnotetext{
${ }^{4}$ Other states (Illinois and Vermont) have established voluntary standards, while still others are considering enacting obligatory RPS policies. A RPS policy has also been considered (but not adopted) by the U.S. Congress.
} 
Commission on Energy Policy (2004) and the Pew Center on Global Climate Change (2004) showed how EE was an important short-term strategy for reducing carbon emissions. Similarly, an analysis by Pacala and Socolow (2004) presented fifteen "stabilization wedges" to reduce carbon emissions, four of which involved EE improvements; the authors asserted that improvements in EE probably offered the greatest potential to provide wedges. As a result, there has been a paradigm shift in how energy policymakers and utility regulators see the world: their "energy lens" has changed to a "climate change lens:" all energy policies and programs are now viewed as solutions to reducing GHG emissions.

Recently, local and state government and Congress have been fervently developing policies and programs to reduce emissions. In the Northeast, states are developing a carbon cap and trade system under the Regional Greenhouse Gas Initiative (RGGI). In California, Governor Schwarzenegger established new goals for GHG emissions, announcing in an executive order that his goal is "by 2010, to reduce GHG emissions to 2000 levels; by 2020, reduce GHG emissions to 1990 levels; by 2050, reduced GHG emissions to 80\% below 1990 levels.” The goal of achieving 1990 GHG emission levels by 2020 was subsequently incorporated into law when the State legislature passed, and the governor signed, AB 32 in September 2006. In addition, SB 1368, signed into law at the same time, requires that the CPUC and the Air Resources Board establish GHG emission performance standards for all base-load generation. This legislation increases the importance of both RE and EE efforts.

\section{Evaluation Issues}

The evaluation issues that evaluators of EE programs have addressed in the last thirty years will continue to be relevant in the evaluation of DR, RE, and CC mitigation programs. However, certain issues will be emphasized (or de-emphasized) more than others, and new issues will need to be evaluated. Due to paper size limitations, we can only highlight some of the key issues.

\section{Baselines \& Additionality}

In the evaluation of EE programs, the identification and measurement of a baseline is critical for the calculation of energy savings. One needs to know what is likely to have happened to energy use if the program had not been implemented. For example, for calculating the energy 
savings from the installation of an EE appliance, the baseline may include applicable state and/or Federal EE appliance standards, existing equipment efficiency, or common replacement practices. The measurement of a well-matched non-treatment comparison group over time can also provide a reasonable baseline.

Baselines will continue to play a critical role in the evaluation of DR and RE programs. In DR programs, the customer's expected load absent the DR request is the customer's baseline. Various methods of baseline calculation are available: e.g., load averaging of similar days, using regression analysis, or making adjustments to reflect certain factors such as weather. For RE programs, the calculation of the baseline is straightforward: assuming there has been little RE development in the area, one can assume a baseline of zero energy production. If RE programs become successful and increased market penetrations are achieved, then the calculation of the baseline will be more challenging.

It is important that baselines need to be consistently defined. In a review of EE protocols, baseline conditions were found not to be always consistently defined when estimating savings (Michals and Titus 2006). For example, in new construction programs, the baseline was typically defined as standard practice (i.e., the state's current building energy code or common practice): therefore, the baseline varied from state to state depending on what energy code is in place and typical practice in the area. And in the case of early replacement/retirement retrofit programs, some states defined the baseline as either (a) the existing measure or technology, (b) standard practice or what was required under the state's building energy code, or (c) a blend of existing technology and current standard practice/code.

For CC programs, it is important to know if a program (or project) reduced GHG emissions that were additional to any that would have occurred in the absence of the project activity. At the international level, the Clean Development Mechanism's (CDM) Executive Board has developed rules that developers must follow to demonstrate the additionality of their proposed projects (CDM 2004). ${ }^{5}$ These rules are consistent with the methods used to estimate

\footnotetext{
5 The Kyoto Protocol includes two project-based mechanisms for activities across countries. Article 6 of the Protocol allows for joint implementation (JI) projects between developed (Annex I) countries: i.e., project-level trading of emissions reductions can occur among countries with GHG emission reduction commitments under the Protocol. Article 12 of the Protocol provides for a CDM that allows legal entities in the developed world to enter into cooperative projects to reduce emissions in the developing world for the benefit of both parties. Developed countries will be able to use certified emissions reductions from project activities in developing countries to
} 
gross and net savings in the evaluation of EE programs, and it is expected that these rules will be used for demonstrating additionality for an international emissions trading scheme. Thus, for state and national cap and trade programs, if credits are issued and credits are allowed to be bought and sold at the international level, then additionality will need to be rigorously addressed. To some degree, additionality is already being addressed at the state level where EE baselines have been identified and free riders have been evaluated, as described in the next section.

\section{Adjustments to Gross Energy Savings: Free Riders and Program Spillover}

In EE programs, gross savings quantify the estimated change in energy consumption or demand that results directly from EE measures taken by participants in the program, regardless of why they participated. Through evaluations and other studies or assumptions about "free riders” ${ }^{6}$, gross savings are adjusted to determine net savings, which reflect only EE savings that can be attributed to the program. Net savings are used for the purposes of measuring program cost-effectiveness and awarding program administrators for performance. In the Northeast, net savings are also used to inform projected energy and demand reductions in regional system planning (Michals and Titus 2006). In contrast, in the Northwest, gross savings are used for forecasting purposes on the basis that they should reflect energy efficiency investments regardless of who pays (i.e., including free riders) (Keating 2007). In a review of EE protocols in the Northeast, states had different rules on what adjustments were applied to gross savings to calculate net savings (Michals and Titus 2006).

In addition to adjusting for free riders, gross savings can also be adjusted up or down to reflect energy and demand savings due to spillover (participant or non-participant). Participant spillover represents the additional EE actions that program participants take outside a program as a result of having participated in the program. Non-participant spillover represents the changes in the energy use of non-participants as a result of the program. In a review of EE protocols in the Northeast, states varied in their use (or non-use) of spillover effect as part of their calculation of net savings (Michals and Titus 2006).

contribute to their compliance with GHG targets. The Kyoto Protocol also provides for an emissions trading (ET) scheme (Article 17). The CDM rules are the most advanced of the three provisions, as the design of JI and ET is still being developed.

${ }^{6}$ In energy efficiency projects, it is possible that the reductions in energy use are undertaken by participants who would have installed the same measures if there had been no project. These participants are called "free riders." The savings associated with free riders are not truly "additional” to what would occur otherwise. 
Adjustments to gross savings are not examined in DR programs, since customers cannot take advantage of the benefits of these programs without participating in the program itself. For reporting purposes for the Forward Capacity Market, the New England Public Utility Commissioners voted to provide gross EE savings rather than net, for the following reasons: (1) consensus on the use of spillover in the definition of net savings did not exist, (2) other demand providers reported gross EE savings, and (3) there was concern that spillover measurements would not withstand the rigor of M\&E requirements. However, as these programs become more available, free ridership may become more of an issue. Also, it will be relatively easy to evaluate non-participant spillover in the future, since customers will need to apply to these programs to receive the special incentives. As mentioned previously, renewable energy is still relatively "new" and one expects little free ridership. As in the case with DR programs, as renewable energy programs become more widespread (e.g., via RPS or voluntary carbon programs), measurement of free ridership and program spillover effects may become more of an issue.

The future relevance of free ridership is under question, especially with the recent interest in CC. Under a "market transformation" perspective where one is concerned about progress being made towards an EE market/society, one may not be concerned about free riders, but are concerned about market share and the EE activities of other participants. In fact, the more free riders, the better. Furthermore, it is expected that EE programs will benefit from the increased awareness of how EE efforts reduce GHG emissions. For example, emission tax or trading schemes may provide a financial incentive to invest in EE measures. Similarly, increased media attention to energy issues may change behavior patterns and generate demand for EE measures. This increased coverage is readily apparent in the popular media and financial publications, and behavior in residential customers may already have changed as a result. And as business sectors try to reduce their GHG emissions, there will be increasing attention to EE. Hence, more residential and business customers are becoming more sensitized and better educated about EE and will be more interested in reducing their own energy use. As a result, it will be much more challenging to have cost-effective programs based on net energy savings that account for free riders. In summary, if one believes that energy forecasts cannot account for all of the EE improvements that would occur naturally, then it may be best to focus on estimating gross energy savings and devote less evaluation resources to quantifying the number of free riders. 


\section{Reliability, Uncertainty, and Precision}

Because of the difficulties and uncertainties in estimating energy savings and reduced emissions, the level of precision and confidence levels associated with the measurement of savings need to be identified (Vine and Sathaye 1999). The precision of measurements and results can be demonstrated in one of two ways: (1) quantitatively, by specifying the standard deviation around the mean for a bell-shaped distribution, or providing confidence intervals around mean estimates; or (2) qualitatively, by indicating the general level of precision of the measurement (e.g., low, medium or high). The EE program evaluation community has responded to the uncertainty challenge by conducting evaluation studies using rigorous sampling techniques that measure and report savings with a specific level of confidence (e.g., the energy savings were 1,000 kWh plus or minus $20 \mathrm{kWh}$ at a 90\% confidence level). California's measurement and evaluation protocols contain advice on sampling as well as choosing the appropriate methodology depending on the level of rigor that is required (CPUC 2006).

A related key concern of resource procurement planners and independent system operators is whether EE is a reliable resource. Reliability is critical when making sure there is sufficient supply to meet society's needs. On the reliability issue, it is important to place the reliability and uncertainty of EE in the proper context (Vine et al. 2007). While we recognize that there are uncertainties associated with EE as an energy resource, there are also uncertainties on the supply side. Therefore, any assessment of the reliability of EE as a resource needs to be done in the context of how these concepts come into play in planning and operating a utility system. For example, critics of EE sometimes seek to portray energy efficiency as inherently uncertain and unreliable, and supply side resources as well known and dependable. That portrayal, simply put, is false. In fact, substantial uncertainties exist regarding the planning and implementation of every utility system resource (e.g., delays in the construction of power plants, increasing costs of power plant construction, variable fuel prices, and the costs and timing of transmission and distribution projects). Since we expect that critics may question the value of GHG emissions reduction from EE measures, it is important to remind people about the risk-reducing value of EE. First, and most obviously, EE avoids fuel-cost risk entirely, which is a significant advantage in this new era of high and volatile energy prices. Second, EE is not dependent on a single high capital cost project like a power plant. Rather, the EE resource is composed of a large number of 
relatively small incremental cost projects. Finally, EE is a very flexible resource that can be acquired in larger or smaller increments in response to utility system needs, thereby dramatically reducing the risk of over-building or under-building utility system resources.

DR programs face the same reliability concerns as EE, especially for dispatchable DR, which is considered to be a supply-side resource for purposes of calculating resource adequacy requirements. Can resource planners count on DR at critical periods (e.g., in time of peak demand)? ISO New England's (ISO-NE) measurement and verification (M\&V) manual for demand resources requires that project sponsors have to show how they will meet or exceed the statistical precision and accuracy standards specified in ISO-NE’s M\&V manual (ISO-NE 2007). Many renewable energy systems (e.g., wind) have intermittency problems, and, therefore, have the same reliability and uncertainty concerns, but with less concern about precision: for example, the electricity generation from a photovoltaic system is equal to the output of the system, and this can be directly measured. It may be best to look at DR and RE from a portfolio perspective, as one of several resource options that can be called upon in times of need. A probability assessment could be conducted for DR and RE to see which resources could be used and at what time period.

GHG emissions reductions will confront the same challenges with respect to uncertainty, and the precision issue may be greater than the EE savings uncertainty, depending on the emissions factor that is used (e.g., a default emissions factor (emissions allocation rate), a factor based on a utility dispatch model, or something in between) (Vine and Sathaye 1999; USEPA 2007). For example, the dispatch model is generally considered the most rigorous means of quantifying displaced emissions rates, however, it is labor intensive, expensive, and generally difficult for non-experts to evaluate. On the other hand, the advent of credits for carbon reductions and the monetization of those credits may create an environment where precision is very important to buyers and sellers of the credits.

In conclusion, in most situations, uncertainty estimates will usually require expert judgment. If one is unsure of the final answer, one can try to discount the results (for energy savings, demand reduction, or GHG emissions reduction) (Vine et al. 2003). On the other hand, discounting may discourage program participation if discounting rates are set too high, and discounting may lead to gaming - e.g., savings may be overestimated by $20 \%$ if the sponsors 
know that their savings will be discounted by $20 \%$. Thus, as an alternative, one might want to set minimum uncertainty and reliability standards, or limits of acceptable accuracy (USEPA 2007). If the standard is met, there is no discounting. If the standard is not met, then the project is not allowed.

\section{Persistence}

Persistence reflects whether or not an installed EE measure is still installed (measure retention) over the anticipated lifetime of the measure, or can take into account changes in how the measure is used and how savings may change over time due to technology degradation. Thus, a persistence study measures changes in the net impacts that are achieved through installation/adoption of program-covered measures over time, and these changes include retention and performance degradation. Many past persistence studies were unable to provide results that were significantly different (statistically) from the ex-ante results, so that most of the current ex-post effective useful lives (i.e., measure lifetimes) are the same as the ex-ante estimates (CPUC 2006). Besides finding relatively high retention rates in most cases, a consistent and important finding from these studies was that a longer period of time is needed for conducting these studies, so that larger samples of failures are available, and so that technology failure and removal rates can be better documented and used to make more accurate assessments of failure rate functions. California's measurement and evaluation protocols address persistence in the Effective Useful Life Evaluation Protocol (CPUC 2006). But persistence is not consistently accounted for in other states. For example, in a review of EE protocols in the Northeast, some states applied an explicit persistence or retention factor as part of a net savings algorithm, while in other states "persistence" was captured in the assumed measure life and, therefore, while accounted for, was not readily transparent (Michals and Titus 2006).

Persistence may not be an issue for DR programs, since the impacts from many of these programs are short-lived: the reduction of energy use at critical peak times of the day (e.g., 3 PM to $6 \mathrm{PM}$ on a Summer weekday). However, persistence is important for RE programs, particularly if one is evaluating programs and technologies from a lifecycle cost analysis. For example, the maintenance of a photovoltaic (PV) system is critical, so that the PV load does not decrease over time. This is an issue, for example, in California's Central Valley where dust can form a blanket over the PVs and reduce their performance. 
For CC programs, the persistence issue is addressed in the determination of the GHG emission credits in both voluntary and mandatory carbon markets. Although some EE measures may have long lifetimes (e.g., 20-30 years), the credits representing these reductions may be of limited duration (e.g., 7 years). If a CC program wanted to extend longer lifetimes for certain measures, a persistence study would need to be conducted. Similarly, for shorter lifetime measures (e.g., less than 7 years), a persistence study might be needed to see if the carbon credit should be discounted (see below). Because of the effort needed in conducting persistence studies, the value of carbon credits would need to be sufficiently high to warrant such a study.

\section{Evaluation Approaches and Methods}

Over 1,000 evaluation studies have been prepared in the U.S., and the energy savings of EE programs have been closely reviewed in contested regulatory hearings in dozens of states. In the twenty first century, we now have an entire energy evaluation industry and dedicated professional organizations continuing to enhance the methods, standards, and conduct of energy program evaluation. The results of this work are published in peer-reviewed journals and conference proceedings. A wide range of evaluation methodologies has been developed and refined over the past thirty years to estimate energy savings with acceptable levels of precision. These evaluation techniques have featured many sophisticated methods to rigorously assess EE impacts. We expect that many of these methods will be used in the evaluation of the impacts of DR, RE, and CC programs. ${ }^{7}$ Below, we mention three types of evaluation approaches that should be considered when evaluating DR, RE, and CC programs.

\section{Impact Evaluation}

The evaluation of (gross and net) energy savings ( $\mathrm{kWh}$, $\mathrm{kW}$, therms) has been the centerpiece of the evaluation of EE program evaluation for many years, and the methods for evaluating these impacts are described in guidelines at the state (e.g., the California measurement and evaluation protocols - CPUC 2006), national (e.g., the U.S. Department of Energy's

\footnotetext{
${ }^{7}$ A protocol has been developed for evaluating RE as part of the IPMVP (see below), the California Public Utilities Commission is in the process of developing an evaluation protocol for DR, ISO New England has developed a manual for the evaluation of DR programs (ISO-NE 2007), and a protocol is being developed for the evaluation of CC programs (USEPA 2007).
} 
International Measurement Performance and Verification Protocol (IPMVP) ${ }^{8}$ ), and international (e.g., International Energy Agency’s (IEA) Evaluation Guidebook (IEA 2005)) levels. The methods can vary depending on many factors, such as budget, the level of rigor and precision required, program type, and type of EE measure. Some programs may simplify their evaluation approach by basing the savings on stipulated or deemed savings. ${ }^{9}$ Alternatively, billing analysis or building energy computer simulations may need to be run (for complex EE systems, new construction, etc). Many organizations rely on the IPMVP to determine which methods to use. Interest in evaluating peak demands from EE measures is gaining attention as greater weight is being placed on capacity $(\mathrm{kW})$ than energy $(\mathrm{kWh})$ savings from EE measures. However, a greater understanding and accurate quantification of the peak demand impacts from EE measures remains (York et al. 2007).

The methods and approaches used for evaluating EE impacts can be transferred to the evaluation of DR, RE, and CC impacts, although with some differences. For DR programs, the time period of analysis is different than typically used for EE programs. For example, the analysis of energy savings from EE programs typically occurs over longer periods of time: e.g., kWh saved per year; sometimes there is interest in monthly or daily analysis. However, for DR programs, hourly analysis is needed. DR program impacts may vary according to the circumstances of the event, such as temperature, humidity weather, day of the week, time of day, location, and type of system emergency (see ISO-NE’s M\&V Manual - ISO-NE 2007; Violette and Hungerford 2007; Summit Blue and Quantum Consulting 2006). For RE programs, the focus is on energy production, rather than on energy savings. However, the measurement options are basically the same (USEPA 2007). For long-term procurement planning, the evaluation of RE programs may place more emphasis on capacity $(\mathrm{kW})$ than energy $(\mathrm{kWh})$ savings.

For CC programs, the GHG impacts from EE programs can be calculated in one of several ways (Vine and Sathaye 1999; USEPA 2007). Once the energy savings have been calculated, GHG emissions reductions can be calculated in one of two ways: (1) if emissions reductions are based on fuel-use or electricity-use data, then default emissions factors can be used, based on utility or nonutility estimates; or (2) emissions factors can be based on generation

${ }^{8}$ The Efficiency Valuation Organization (EVO) is now responsible for maintaining and updating the IPMVP (www.evo-world.org).

9 Stipulated data may be taken from engineering energy savings analyses, efficiency program work-papers, secondary research, engineering references, manufacturers' catalog data, and/or on-site survey data. 
data specific to the situation of the project (e.g., linking a particular project on an hourly or daily basis to the marginal unit it is affecting). In both methods, emissions factors translate consumption of energy into GHG emission levels (e.g., tons of a particular GHG per kWh saved). In contrast to default emission factors (method \#1), the advantage of using the calculated factors (method \#2) is that they can be specifically tailored to match the EE characteristics of the activities being implemented by time of day or season of the year. For example, if an EE project affects energy demand at night, then baseload plants and emissions will probably be affected. Since different fuels are typically used for baseload and peak capacity plants, then emission reductions will also differ.

The calculations, however, become more complex (but more realistic) if one decides to use the emission rate of the marginal generating plant (multiplied by the energy saved) for each hour of the year, rather than the average emission rate for the entire system (i.e., total emissions divided by total sales). For the more detailed analysis, one must analyze the utility's existing expansion plan to determine the generating resources that would be replaced by saved electricity, and the emissions from these electricity-supply resources. Thus, one would establish a baseline (current power expansion plan, power dispatch, peak load/base load, etc.), select a monitoring domain, conduct a monitoring option, measure direct emission reductions (e.g., reductions occurring at the neighboring power plant to lower demand), measure indirect emissions (e.g., modification in the power system due to lower output at the neighboring plant), and calculate net carbon reductions.

One would have to determine if the planned energy-efficiency measures would reduce peak demand sufficiently and with enough reliability to defer or obviate planned capacity expansion. If so, the deferred or replaced source would be the marginal expansion resource to be used as a baseline. This type of analysis may result in more accurate estimates of GHG reductions, but this method will be more costly and require expertise in utility system modeling. In addition, this type of analysis is becoming more difficult in those regions where the the supply of energy may come from multiple energy suppliers, either within or outside the utility service area.

The decision on which methodology to use will depend on project size (e.g., kWh, kW, carbon credits requested, project expenditures) or relative project size (e.g., MW/utility service 
MW). It is up to the evaluator to decide on the best method for the project. Certain thresholds may need to be developed. If a project is of a certain relative magnitude (e.g., a project is $50 \mathrm{MW}$ and the utility's service area is $400 \mathrm{MW}$ ), the evaluator would probably select the second method above.

\section{Market Effects Evaluation}

Market effects evaluations document the various market changes that affect the way energy is used within a market and estimate the energy and demand savings associated with those changes that are induced by sets of program or portfolio interventions in a market (CPUC 2006). This is very useful for examining the transformation of markets. In California, the CPUC's measurement and evaluation protocols include a market effects protocol. This protocol does not apply to the measurement of individual program-level market effects or direct program savings typically used for program-level cost-effectiveness assessments and refinement decisions. Rather the focus of the market effects evaluation is at a market level in which may different energy efficiency programs can operate. This protocol can be used for evaluating program-induced market changes that could be missed or double counted if measured program by program. One of the key tools in market effects evaluation is a logic model that builds a concise description of a program's performance (Dowd et al 2005; CPUC 2006). Logic models are two-dimensional graphics and supporting text that describe a sequential set of activities in one dimension and the performance spectrum, i.e., the resources required for these activities, the outputs, the target audience, the short-/intermediate- and long-term outcomes in the other dimension. The logic model and market theory are used to guide the market effects evaluation and then to develop a list of indicators for tracking market effects.

The key market indicator in the market effects evaluation of EE programs is the value of energy savings from sales and/or market share changes for targeted efficient measures. Other related indicators include changes in awareness, intention to purchase, stocking practices, product availability, prices, and willingness to invest in EE. This type of analysis could also be used for DR, RE and CC programs. For DR programs, it would be useful to see how the program has changed the way energy is being used in buildings (e.g., metering systems, energy management systems, purchase of smart meters and appliances, changes in behavior during peak hours, etc.). For RE programs, it would be useful to see how the program has changed the type of 
$\mathrm{RE}$ system that is being installed, sales of $\mathrm{RE}$ technologies, the supply of green pricing options and renewable energy certificates, etc. For CC programs, it would be useful to see how all EE programs' impacts have changed the value and supply of carbon credits. For all programs, a market effects evaluation will be useful for identifying market infrastructure development needs that can contribute to program success, the barriers limiting program success, and program design and implementation strategies that are market focused.

\section{Process Evaluation}

Process evaluation is often critical to the successful implementation of cost-effective and cost-efficient EE programs. Process evaluations identify improvements or modifications to a group of programs, individual programs or program components, that directly or indirectly acquire or help acquire, energy savings in the short-term (resource acquisition programs) or the longer-term (education, information, advertising, promotion and market effects or market transformation efforts). Many process evaluations of EE programs have been conducted (especially in California) for evaluating the efficiency of program implementation as well as for identifying program enhancements and improvements. While process evaluation is not a required evaluation activity in California, it is part of the California evaluation protocols (CPUC 2006).

Process evaluation has been used in DR program evaluation for evaluating program participants and nonparticipants, identifying program improvements (e.g., audits of customers), and for identifying training needs for vendors for delivering program services. In addition, a new initiative was implemented in early 2007 to advance the scientific understanding of customer behavior related to DR, hopefully leading to the increased adoption of DR programs and activities and improved process evaluations of DR programs (PIER DRRC 2007). Similarly, process evaluation could be used for developing a better understanding of barriers to participation in RE programs and of customer behavior related to RE, as well as for program improvement. And for CC programs, process evaluation could be used to identify which strategies provide the most significant impact for GHG emissions reduction, as well as for program improvement.

One important subset of process evaluation is the development of program theory (and logic modeling) - see Market Effects Evaluation section. An important aspect of this type of 
analysis is the examination of the social and behavioral issues affecting program participation as well as investment in EE. What makes people invest in technologies and EE measures? What are their business models? How do tariffs and rates affect their decisions? What about cost savings what is their payback level? It can also be used for market segmentation analysis and potential savings. This could be used in the evaluations for all programs.

\section{Expansion and Extension of Evaluation Protocols}

Evaluation protocols are needed to measure, verify, and report EE and DR savings, RE generation, and GHG emissions reductions to assist program managers, resource planners and forecasters, and utility regulators. As referred to previously, several key protocols and guidelines have already been developed: nationally (IPMVP; USEPA 2007), regionally (New England NE-ISO 2007), and at the state level (e.g., California - CPUC 2006). However, differences in current evaluation protocols can either under- or over-state the overall regional, state, or local impact of EE to reduce power plant emissions, potentially resulting in shortfalls in meeting emission reduction targets. Thus, there is a need for common, consistent and transparent evaluation protocols (Michals and Titus 2006). These protocols will ensure that the measurement, verification, and reporting of savings from EE, DR, and RE are: reasonable and defensible, providing sufficient credibility and certainty; transparent, based on documented sources; and consistent, so that savings (and generation) can be tracked and readily aggregated. Some progress has already been made: in the Northeast, the NE-ISO M\&V Protocol is being used by the states in New England for evaluating EE and DR programs (NE-ISO 2007). In California, public agencies, utilities, environmental and other groups have started a project to support EE evaluation, measurement, and verification (EM\&V) best practices in California, nationally and internationally (Schiller 2007). The project includes a number of planned activities such as a Model EM\&V Guideline (to be prepared in conjunction with the National Action Plan for Energy Efficiency) and an EE climate change mitigation project protocol. In sum, the EE protocols from the state level are being expanded to include to cover a larger scope of public purpose and CC programs. It will be interesting to see if multiple sets of protocols will lead to problems in consistency in EM\&V and the reporting of results. A national protocol may need to be developed to provide this consistency, so that GHG credits from EE programs can be traded across states. 


\section{Integrating Policies: Breaking Down the Silos}

Evaluation professionals are currently caught in a policy dilemma when evaluation issues are being addressed in multiple policy arenas ("silos”): e.g., before addressing the reduction of GHG emissions from RE programs, one has to wait and see how the reduction of GHG emissions from EE programs are being addressed by utility regulators. ${ }^{10}$ Consequently, regulatory policies are needed for integrating EE programs with DR, RE, and CC programs (and probably other types of programs, too - e.g., water efficiency programs ${ }^{11}$ ). This will not be easy, since power, influence, and budgets are associated with individual silos. Different stakeholder groups are associated with each silo, so they may either be disinterested in the other silos, or interested in not seeing their influence possibly diminished with the integration of the silos. Finally, in some cases, there may be cultural differences with different languages, perspectives, and needs. The differences between EE and air quality communities provide a good example (Vine 2003). Energy personnel assume that reductions in energy use reduce or displace new or existing energy production. In their minds, these hypothesized reductions or displacements of energy production translate directly into actual emission reductions. Historically, they have not concerned themselves with demonstrating where, when, or whether emissions reductions occur at actual, specified emissions sources. In contrast, air quality personnel focus on emissions sources to determine allowable emissions levels and emissions reductions. For them, reducing electricity demand in a non-attainment area does not necessarily translate into an emissions reduction within the airshed. For some air quality regulators, only reduced activity or lowered emissions rates at power plants located within the non-attainment area can qualify as emissions reductions in air compliance plans. This is reinforced because the emissions measurements are made at the point of production, and not at the point of ultimate use. In sum, what is measured and how it is accounted for in air compliance plans is a pivotal issue that must be resolved before air quality and energy officials can begin to identify new opportunities for EE improvements within air quality compliance strategies.

\footnotetext{
${ }^{10}$ Another example is determining avoided cost-related issues across multiple proceedings, where methods and inputs for specific applications of avoided costs need to be consistently applied.

11 Although not discussed in this paper, another area receiving increased regulatory attention is the inclusion of "embedded energy savings" from water efficiency projects (by conserving water, using less energy-intensive water, or making current delivery and treatment systems more efficient) in utility EE programs and goals. California IOUs are planning pilot projects to explore the potential for future programs to capture water-related embedded energy savings.
} 
Through inertia alone, an integrated solution to this vexing problem may go unresolved. However, there are signs that this inertia may be breaking down. First, and most significantly, climate change is the driving force that is forcing all of the policy arenas to consider the impacts of their policies on the reduction of GHG emissions, as well as the impacts of a GHG reduction policy on their policies. In addition, several issues have surfaced that EE program managers and utility regulators are starting to consider. For example, what are the GHG emissions reductions from $\mathrm{kWh}$ and $\mathrm{kW}$ savings from EE programs? Next, how does the preferred method get incorporated into reporting requirements and protocols? And should the value of GHG emission reductions (e.g., as reflected in the monetization of carbon credits) be included in costeffectiveness tests (e.g., like the total resource cost test) ${ }^{12}$ Finally, the ownership of credits from reducing GHG emissions will elevate the integration issue: for example, if public goods charge funds (paid by utility ratepayers) are spent on utility programs for providing financial incentives to households for investing in EE, and these savings result in GHG emission reductions, who will own the carbon credits for the GHG emission reductions? Should it be based on economic criteria ("who pays the most, gets the most"), or on policy considerations (e.g., the impact on the promotion of energy efficiency)? In January 2007, the CPUC ruled in D. 07-01-018 that they would allow renewable distributed generation system owners to retain $100 \%$ of their renewable energy credits (RECs), and that utilities would not be able to count the output of renewable distributed generation facilities that have received ratepayer incentives toward the utility's renewable portfolio standard obligations. Other states have made other determinations through either regulatory or legislative authorities (Holt et al. 2006). One might expect a similar decision favoring consumers (rather than utilities) when deciding on the ownership of carbon credits when public benefit funds are involved. ${ }^{13}$ Alternatively, this could be a negotiated contract issue between the provider of financial assistance (e.g., a utility) and the recipient of the funds (e.g., household or government agency). If a national emissions trading program were to be developed, then the ownership issue would need to be resolved at the federal level.

Second, there are areas of overlap where the different policy arenas are already collaborating and coordinating their efforts. This is especially true for the EE and RE policy

\footnotetext{
${ }^{12}$ Some states (like California) have externality values reflecting environmental attributes that are used for resource procurement decisions. This currently does not include the monetary value of carbon reductions or credits.

${ }_{13}$ Twenty states have established public benefit funds to pay for public benefit programs; see aceee.org/energy/pbf.htm.
} 
arenas. At the program and building level, zero-energy homes (or near-zero energy homes), which produce as much electricity (or not quite as much total electricity) as the residents consume, integrate EE and RE in the construction of new homes (Vang and Hammon 2007; Anderson et al. 2007). At the policy level, builders of solar homes are required to exceed the current State's EE standards' (Title 24) performance by 15\% under California's New Solar Homes Partnership. And at the corporate level, the Pacific Gas \& Electric (PG\&E) Company has added a requirement for making the linkage between EE and RE even stronger: as of January 1, 2007, all existing residential and commercial customers must have an EE audit conducted on their existing home or building if they choose to apply for a solar incentive (Barros and Segerstrom 2007). Other areas of opportunity for integrating EE and RE include (1) the development of markets for tradable EE and RE credits, (2) EE resource standards and RE portfolio standards, and (3) the allocation of public benefits funds for EE and RE (Prindle et al. 2007).

Third, some organizations are already integrating EE, DR, RE, and CC. For example, PG\&E markets a package of services to customers in the following order: EE, DR, and RE. Utility customers can also participate in a carbon offset program ("ClimateSmart”) where they would pay more on their monthly utility bills to offset GHG emissions so that they could become “carbon neutral.” Customers would be charged about $\$ 4.30$ a month, based on the household's or business's energy usage, and PG\&E would use the money to offset the carbon dioxide, starting with projects to replant trees and to buy and preserve forests in California. Finally, local governments address these issues, often separately, but more recently as an assemblage when deliberating over the design and implementation of sustainable cities and integrated solutions to EE, RE, wastewater, etc.

\section{Conclusions}

This paper has shown that the EE EM\&V community has addressed and provided guidance on key evaluation issues that will need to be addressed in the evaluation of DR, RE, and CC programs: e.g., baseline and additionality, measurement of gross energy savings, adjustments to gross savings (free riders and program spillover), reliability, uncertainty and precision, and persistence. Similarly, EE evaluation approaches (impact evaluation, market effects evaluation, and process evaluation) will also be useful for evaluating DR, RE, and CC 
programs. EE evaluation protocols are being expanded to evaluate DR, RE, and CC programs (e.g., by the CPUC, USEPA, and the National Action Plan for EE). Finally, policy mechanisms are being developed for integrating EE, RE, DR and CC programs: (1) GHG emissions reductions will need to come from EE, RE, and DR programs using similar and consistent EM\&V methodologies and protocols that address the key evaluation issues identified above; (2) utilities are marketing EE, RE, DR and CC to customers; and (3) EE and RE policies are being more closely coordinated via ZENH, the development of markets for tradable EE and RE credits, EE resource standards and RE portfolio standards, and the allocation of public benefits funds for EE and RE.

The EE program evaluation community is at a critical juncture. DR, RE, and CC programs are becoming more widespread, and they will need to be evaluated by professional evaluators in order for the results of these programs to be credible. This opportunity will also bring challenges and frustrations as the different “energy cultures” learn from each other. One of the key issues will be who makes the final decision on key policies and technical issues at the state, regional, federal, and international levels, and how will these policies and agreements be coordinated? At the same time, the policy regulatory environment will need to change and be adaptive for integrating the policy silos that are interdependent but currently treated separately. Policy and regulatory initiatives are needed for fostering the integration of these silos, so that a more coherent and cohesive strategy can be developed for responding to the threat of climate change and for creating a more sustainable society.

\section{Acknowledgements}

I want to thank the following people for providing comments on the ideas contained in this paper: Nick Hall, Jan Hamrin, Ken Keating, Bruce Mast, Rob Rubin, Steve Schiller, Elizabeth Titus, Dan Violette, and Carol White.

\section{References}

Anderson, R., R. Hammon and M. Keesee 2007. "Maximizing the Benefits of Zero-Energy Homes,” Home Energy, Solar \& Efficiency Special Issue, pp. 42-47. 
Barros, C. and C. Segerstrom 2007. "The California Solar Initiative," Home Energy, Solar \& Efficiency Special Issue, pp. 6-8.

[CDM] Clean Development Mechanism, Executive Board 2004. "Sixteenth Meeting (Oct. 21, 2004) Report.” CDM-EB-16. Available at: cdm.unfccc.int/index.html.

[CPUC] California Public Utilities Commission 2006. California Energy Efficiency Evaluation Protocols: Technical, Methodological and Reporting Requirements for Evaluation Professionals. San Francisco, CA: California Public Utilities Commission.

[CPUC] California Public Utilities Commission 2007. Order Instituting Rulemaking, Rulemaking 07-01-041, Jan. 25, 2007. San Francisco, CA: California Public Utilities Commission.

Chen, C., R. Wiser, and M. Bolinger 2007. Weighing the Costs and Benefits of State Renewables Portfolio Standards: A Comparative Analysis of State-Level Policy Impact Projections, LBNL-61580. Berkeley, CA: Lawrence Berkeley National Laboratory.

Dowd, J., G. Jordan, J. Reed, and E. Vine 2005. “Are Federal Energy Technology Programs Assessing the 'Magic in the Middle'?” Proceedings of the 2005 International Energy Program Evaluation Conference, Brooklyn, NY.

Geller, H. 2006. "Catching Up: Progress with Utility Energy Efficiency Programs in the Southwest," Proceedings of the 2006 Summer Study on Energy Efficiency in Buildings. Washington, D.C.: American Council for an Energy Efficient Economy.

Holt, E., R. Wiser, and M. Bolinger 2006. Who Owns Renewable Energy Certificates? An Exploration of Policy Options and Practice. Report LBNL-59965. Berkeley, CA: Lawrence Berkeley National Laboratory. 
Hopper, N., C. Goldman, and J. Schlegel 2006. Energy Efficiency in Western Utility Resource Plans: Impacts on Regional Resource Assessment and Support for WGA Policies. Report LBNL-58271. Berkeley, CA: Lawrence Berkeley National Laboratory.

[IEA] International Energy Agency, Implementing Agreement on Demand-Side Management Technologies and Programmes 2005. Evaluating Energy Efficiency Policy Measures \& DSM Programmes, Volume 1, Evaluation Guidebook. Paris: International Energy Agency.

[IPCC] Intergovernmental Panel on Climate Change 2007. Climate Change 2007: The Physical Science Basis, Summary for Policymakers. Geneva, Switzerland: Intergovernmental Panel on Climate Change.

[ISO-NE] ISO New England, Inc. 2007. Manual for Measurement and Verification of Demand Reduction Value from Demand Resources. Holyoke, MA: ISO New England.

Keating, K. 2007. "What is the Purpose of Net Savings? A Northwest Perspective," presentation at the $17^{\text {th }}$ National Energy Services Conference, January 31, 2007, Las Vegas, NV.

Kushler, M., D. York, and E. Vine. 2005. "Energy-Efficiency Measures Alleviate T\&D Constraints,” Transmission and Distribution World, 57(4): 32-41.

Michals, J. and E. Titus 2006. "The Need for and Approaches to Developing Common Protocols to Measure, Track, and Report Energy Efficiency Savings in the Northeast,” Proceedings of the 2006 ACEEE Summer Study, pp. 8-179 to 8-190. Washington, D.C.: American Council for an Energy-Efficient Economy.

National Commission on Energy Policy 2004. Ending the Energy Stalemate: A Bipartisan Strategy to Meet America's Energy Challenges. Washington, D.C.: National Commission on Energy Policy. 
[NPPC] Northwest Power and Conservation Council 2005. The Fifth Northwest Electric Power and Conservation Plan. Portland, OR: Northwest Power and Conservation Council.

Pacala, S. and R. Socolow 2004. "Stabilization Wedges: Solving the Climate Problem for the Next 50 Years with Current Technologies.” Science 305: 968-972.

Pew Center on Global Climate Change 2004. The 10-50 Solution: Technologies and Policies for a Low-Carbon Future. Washington, D.C.: Pew Center on Global Climate Change.

Prindle, B., M. Eldridge, M. Eckhardt, and A. Frederick 2007. The Twin Pillars of Sustainable Energy: Synergies between Energy Efficiency and Renewable Energy Technology and Policy. Washington, D.C.: American Council for an Energy-Efficient Economy.

[PIER DRRC] PIER Demand Response Research Center 2007. Research Opportunity Notice DRRC RON-03: Understanding Customer Behavior to Improve Demand Response Delivery in California. Berkeley, CA: Lawrence Berkeley National Laboratory.

Schiller, S. 2007. Personal communication with Steve Schiller (Schiller \& Associates), January 10, 2007.

Summit Blue Consulting, LLC and Quantum Consulting, Inc. 2006. Draft Version 1: Protocols for Estimating the Load Impacts from DR Programs, prepared for Working Group 2 Measurement and Evaluation Committee, Boulder, CO.

[USEPA] U.S. Environmental Protection Agency 2007. Creating an Energy Efficiency and Renewable Energy Set-Aside in the NOx Budget Trading Program: Evaluation, Measurement, and Verification of Electricity Savings for Determining Emission Reductions from Energy Efficiency and Renewable Energy Actions (Draft). Washington, D.C.: U.S. Environmental Protection Agency. 
Vang, S. and R. Hammon 2007. "Energy Efficiency and Solar Electricity Go Hand in Hand,” Home Energy, Solar \& Efficiency Special Issue, pp. 30-35.

Vine, E. 2003. “Opportunities for Promoting Energy Efficiency in Buildings as an Air Quality Compliance Approach,” Energy - The International Journal 28(4): 319-341.

Vine, E. and J. Sathaye 1999. An Overview of Guidelines and Issues for the Monitoring, Evaluation, Verification, and Certification of Energy-Efficiency Projects for Climate Change Mitigation. LBNL-43083. Berkeley, CA: Lawrence Berkeley National Laboratory, Berkeley, CA.

Vine, E., G. Kats, J. Sathaye, and H. Joshi 2003. "International Greenhouse Gas Trading Programs: A Discussion of Measurement and Accounting Issues,” Energy Policy 31(3): 211-224.

Vine, E., M. Kushler, and D. York 2007. "Energy Myth Ten - Energy Efficiency Measures are Unreliable, Unpredictable, and Unenforceable.” In B. K. Sovacool and M. A. Brown (eds.), Energy and American Society - Thirteen Myths. New York: Springer Press/

Violette, D. and D. Hungerford 2007. “Development of California Protocols for Estimating the Load Impacts from DR Programs and Cost-Effectiveness Methods,” Proceedings of the 2007 International Energy Program Evaluation Conference, Chicago, IL.

[WGA] Western Governors' Association 2004. WGA Policy Resolution 04-14: Clean and Diversified Energy Initiative for the West, June 22, 2004. Santa Fe, NM: Western Governors’ Association/

York, D., M. Kushler, and P. Witte 2007. Examining the Peak Demand Impacts of Energy Efficiency: A Review of Program Experience and Industry Practices. Washington, D.C.: American Council for an Energy-Efficient Economy. 